\title{
Medial versus Tympanoplasty in Large Central and Subtotal Perforation - A Prospective Study
}

\author{
Ajoy Khaowas, ${ }^{1}$ Chiranjib Das ${ }^{2}$
}

\begin{abstract}
$\underline{\text { Introduction }}$
Large central and subtotal tympanic membrane (TM) perforations are difficult to repair because of less vascularity of anterior TM than posterior TM and the anterior bony overhang that blocks visualization. Some studies reported very encouraging results with the medio-lateral tympanoplasty in such cases. We have undertaken this study to find out efficacy of this technique in large central and subtotal perforations and to compare the results of medio-lateral with medial tympanoplasty. Materials and Methods

The present prospective study was conducted in the Department of Otorhinolaryngology of a medical college and hospital, West Bengal from January 2013 to December 2014. Patients were alternatively divided into two groups. Medial technique was used in Group I and medio-lateral technique was used in Group II.

$\underline{\text { Results }}$

Each group comprised of 40 patients each. Maximum number of patients in each group was in the age group of 15-25 years. The overall graft uptake rate in this study was $95 \%$ in medio-lateral technique compared to $80 \%$ of underlay technique.

Conclusion

The medio-lateral tympanoplasty is suitable for reconstruction of large central or subtotal TM perforation. It takes advantage of both medial and lateral grafting methods while avoiding their pitfalls.

Kevwords:

Tympanic Membrane Perforation; Tympanoplasty
\end{abstract}

$\underline{\text { ABSTRACT }}$

$\mathrm{O}$ ne of the common sequelae of chronic otitis media is tympanic membrane (TM) perforation. Large central and subtotal tympanic membrane (TM) perforations continue to be one of the greatest problems in tympanoplasty surgery. Repair of these perforations is less likely to be successful as compared to repair of small and posterior perforations. ${ }^{1,2}$ Large central and subtotal TM perforations are difficult to

1 - Department of ENT, KPC Medical College \& Hospital, Kolkata

2 - Department of ENT, Coochbehar Govt. Medical College \& Hospital, Coochbehar

\section{Corresponding author:}

Dr Chiranjib Das

email: chirubata.das.87@gmail.com repair because of less vascularity of anterior TM than posterior TM and the anterior bony overhang that blocks visualization. ${ }^{3}$

There is a greater risk of re-perforation and obliteration of the anterior part of middle ear cavity. ${ }^{4}$ Although the lateral (overlay) technique has a higher success rate for reconstructions of large central and subtotal TM perforations, serious complications may occur. These problems have been managed by a variety of surgical techniques, such as the use of William's microclip, ${ }^{5}$ sandwich graft tympanoplasty, ${ }^{4}$ over-underlay tympanoplasty. ${ }^{6}$ Yet, a still better method is needed to repair anterior and subtotal TM perforations. Jung and Park in 2005 reported very encouraging results with the medio-lateral tympanoplasty, where graft is placed medially to the posterior half of TM perforation and 
laterally to the anterior half of perforation. This method is a hybrid of the medial and lateral tympanoplasty techniques thus takes advantages of both methods. ${ }^{7}$ In light of very encouraging results of medio-lateral tympanoplasty, we have undertaken a prospective study to find out efficacy of this technique in large central and subtotal perforations and to compare the results of medio-lateral with medial tympanoplasty.

\section{Materials and Methods}

The present study was conducted in the Department of Otorhinolaryngology of a medical college and hospital, West Bengal. Eighty six patients of either sex in the age group of 15-50 years suffering from chronic otitis media with large central or subtotal perforation were selected for the study from the outpatient department during the period from January 2013 to December 2014. We selected patients with ear free from active discharge for at least 4 weeks; Air bone gap more than $25 \mathrm{~dB}$ on pure tone audiogram; good cochlear reserve; without any complication; and no focus of infection in nose and throat. Patients with actively discharging ear; hearing loss $>60 \mathrm{~dB}$; marked deviated nasal septum or active sinus disease; history of ear surgery in the past were excluded from the study. Written informed consent was taken from all patients and their guardian. This study was approved by Institutional Ethical Committee. Six patients did not turn in follow up. So they were excluded from the study. These patients were alternatively divided into two groups. Each group comprised of 40 patients. Medial technique was used in Group I and medio-lateral technique was used in Group II.

History of all the patients was documented in detail. All of them were subjected to thorough clinical examination and the otoscopic findings were confirmed by examination of the ear under microscope (EUM). Pure tone audiogram of both ears for air and bone conduction were done. Patients were posted for tympanoplasty operation under local anaesthesia and sedation after doing all routine investigations. Autologous temporalis fascia graft was used in all cases. Also trans-canal technique was used in all the cases.

In medial tympanoplasty technique graft was advanced under the posterior tympanomeatal flap and under the malleus handle to the anterior most extent of the perforation and the edges of the graft was tucked under the margin of drum remnant. Small pieces of the gelfoam were used to overlap the junction of rim and graft circumferentially.

In medio-lateral tympanoplasty technique vertical canal incisions were made at the 12 and 6 O'clock position. The 6 o'clock incision was extended right up to the annulus. The 12 o'clock incision was made down to a few millimetres above the annulus to preserve blood supply because the anterior canal skin was used as the superiorly based flap. Connecting incision was given $3 \mathrm{~mm}$ away from the annulus on posterior canal skin. Posterior tympanomeatal flap was elevated. With a curved round knife, horizontal incision was made in the anterior canal skin. The distance of the anterior horizontal canal incision from the anterior annulus should be about the same as the diameter of the perforation. After the incision the anterior canal skin was elevated medially. The antero-medial canal skin flap was elevated up to the annulus or margin of the tympanic membrane perforation. At the annulus, only the squamous epithelial layer of the tympanic membrane was carefully elevated to the anterior half of the perforation edge, leaving the anterior annulus intact. The temporalis fascia was grafted medially for the posterior half of the perforation and the handle of the malleus and was grafted laterally over the annulus in the anterior half of the perforation. Antero-medial skin was rotated to cover perforation and fascia in a superiorly based flap. Antero-lateral canal skin was replaced. Gelfoam soaked in antibiotic solution was placed lateral to the tympanic membrane. An umbilical tape coated with framycetin ointment was placed in external auditory canal.

Patients were discharged next day with antibiotic, analgesic and antihistaminic. Each case was reviewed at 1 week, 3 week, 2 months, 6 months and 1 year. Postoperative pure tone audiometry was done at 6 months and 1 year.

\section{Results}

Total 80 patients were divided into two equal groups randomly. Medial tympanoplasty was done in Group I and medio-lateral tympanoplasty was done in Group II. 
Table I: Distribution of patients according to size of perforation

\begin{tabular}{|c|c|c|}
\hline \multirow{2}{*}{ SIZE OF PERFORATION } & TOTAL NUMBER OF PATIENTS & HEALTHY VOLUNTEERS \\
\cline { 2 - 3 } & GROUP I & GROUP II \\
\hline Subtotal Perforation & $34(85 \%)$ & $36(90 \%)$ \\
\hline Large Central Perforation & $\mathbf{0 6}(\mathbf{1 5 \% )}$ & $\mathbf{0 4}(10 \%)$ \\
\hline
\end{tabular}

Table II: Relationship between graft take-up and size of perforation

\begin{tabular}{|c|c|c|}
\hline \multirow{2}{*}{ SIZE OF PERFORATION } & TOTAL NUMBER OF PATIENTS & HEALTHY VOLUNTEERS \\
\cline { 2 - 3 } & GROUP I & GROUP II \\
\hline Large & $4 / 6(66 \%)$ & $4 / 4(100 \%)$ \\
\hline Subtotal & $28 / 34(82 \%)$ & $34 / 36(94 \%)$ \\
\hline Overall & $32 / 40(80 \%)$ & $38 / 40(95 \%)$ \\
\hline
\end{tabular}

Maximum number of patients in each group was in the age group of 15-25 years. There were total 44 males and 36 females. All patients in this study had a history of ear discharge in the past, though the ear was apparently dry for at least four weeks before they were taken up for tympanoplasty. In both groups $15 \%$ patients had bilateral ear involvement. Size of the perforation was defined as follows ${ }^{3}$ : Pin-point; Small (smaller than one quarter of the tympanic membrane size); Medium (up to half the size of tympanic membrane); Large (up to

Table III: Follow up examination in Group I

\begin{tabular}{|c|c|c|c|c|}
\hline OTOSCOPIC FINDINGS & 10TH DAY & 3RD WEEK & 2ND MONTH & 6TH MONTH \\
\hline Intact graft & - & $32(80 \%)$ & $32(80 \%)$ & $32(80 \%)$ \\
\hline Anterior sulcus blunting & - & - & - & - \\
\hline Graft lateralization & - & - & - & - \\
\hline Discharge & - & $8(20 \%)$ & - & - \\
\hline Residual perforation & - & $8(20 \%)$ & $4(10 \%)$ & $2(5 \%)$ \\
\hline Graft rejection & - & - & $6(15 \%)$ & $6(15 \%)$ \\
\hline
\end{tabular}


Table IV: Follow up examination in Group II

\begin{tabular}{|c|c|c|c|c|}
\hline OTOSCOPIC FINDINGS & 10TH DAY & 3RD WEEK & 2ND MONTH & 6TH MONTH \\
\hline Intact graft & - & $\mathbf{3 6}(\mathbf{9 0} \%)$ & $\mathbf{3 6}(\mathbf{9 0} \%)$ & $\mathbf{3 8}(\mathbf{9 5 \%})$ \\
\hline Anterior sulcus blunting & - & - & - & - \\
\hline Graft lateralization & - & - & - & - \\
\hline Discharge & - & $04(10 \%)$ & - & - \\
\hline Residual perforation & - & - & $02(5 \%)$ & - \\
\hline Graft rejection & - & - & $02(5 \%)$ & $02(5 \%)$ \\
\hline
\end{tabular}

three quarters the size of tympanic membrane); Subtotal (when only annulus remains). In Group I, 34 (85\%) cases had subtotal perforation whereas six $(15 \%)$ cases had large central perforation. In Group II, 36 (90\%) cases had subtotal perforation, whereas four $(10 \%)$ cases had large central perforation (Table I). In Group I, four $(10 \%)$ cases had medially retracted handle of malleus.

In rest $36(90 \%)$ cases handle of malleus was normal. In Group II, two (05\%) cases had medially retracted handle of malleus; while it was partially necrosed in two $(05 \%)$ cases and normal in $36(90 \%)$ cases. In Group I, four $(10 \%)$ cases had moist the middle ear mucosa. While the middle ear mucosa was normal in $36(90 \%)$

Table V: Average post-operative hearing gain in Group I

\begin{tabular}{|c|c|}
\hline HEARING GAIN & $\begin{array}{c}\text { NUMBER OF } \\
\text { PATIENTS }\end{array}$ \\
\hline $0-5 \mathrm{~dB}$ & $08(20 \%)$ \\
\hline $5-10 \mathrm{~dB}$ & $00(0 \%)$ \\
\hline $10-15 \mathrm{~dB}$ & $20(50 \%)$ \\
\hline $15-20 \mathrm{~dB}$ & $10(25 \%)$ \\
\hline$>20 \mathrm{~dB}$ & $02(05 \%)$ \\
\hline
\end{tabular}

cases. In Group II, two cases had moist middle ear mucosa, rest were normal. Tuning fork test was done in all patients with $512 \mathrm{~Hz}$ frequency tuning fork and results were compared with the audiogram. Based on the pure tone audiogram, hearing loss was classified as mild (24-40 dB), moderate (41-55 dB), moderately severe $(56-70 \mathrm{~dB})$, severe $(71-90 \mathrm{~dB})$ or profound $(>90 \mathrm{~dB}) .{ }^{19}$ In both the groups $95 \%$ of the patients had conductive hearing loss in the range of $25-40 \mathrm{~dB}$ and four patients had a loss of $45 \mathrm{~dB}$. The overall graft take-up rate in group I was $80 \%$ and in group II it was $95 \%$ (Table II). In Group I, discharge was noted in eight patients which was resolved by changes of antibiotic and six patients

Table VI: Average post-operative hearing gain in Group II

\begin{tabular}{|c|c|}
\hline HEARING GAIN & $\begin{array}{c}\text { NUMBER OF } \\
\text { PATIENTS }\end{array}$ \\
\hline $0-5 \mathrm{~dB}$ & $02(05 \%)$ \\
\hline $5-10 \mathrm{~dB}$ & $00(0 \%)$ \\
\hline $10-15 \mathrm{~dB}$ & $14(35 \%)$ \\
\hline $15-20 \mathrm{~dB}$ & $22(55 \%)$ \\
\hline$>20 \mathrm{~dB}$ & $02(5 \%)$ \\
\hline
\end{tabular}


had graft rejection and two had residual perforation (Table III). In Group II, discharge was noted in four patients which was resolved by changes of antibiotic and two patients had graft rejection and two had perforation which was healed with application of chemical cautery (Table IV).

In Group I, the average preoperative A-B gap was $35.5 \mathrm{~dB}$ while the average postoperative A-B gap was $21.5 \mathrm{~dB}$, giving an average postoperative gain of $14 \mathrm{~dB}$. Majority of patients $30(75 \%)$ had a gain in the range of $10-20 \mathrm{~dB}$. Two patients had a gain of more than 20 $\mathrm{dB}(05 \%)$, eight $(20 \%)$ had $0-5 \mathrm{~dB}$ gain. This included two patients with perforation and six with graft rejection (Table V). In Group II, the average preoperative A-B gap was $36.5 \mathrm{~dB}$ with average postoperative gap of 18.5 dB thereby average gain of $17.5 \mathrm{~dB} .36(90 \%)$ patients had a hearing gain of $10-20 \mathrm{~dB}$, while two $(5 \%)$ had gain of more than $20 \mathrm{~dB}$. Rest two (5\%) patients had no gain of hearing. This group comprised of two cases with graft rejection (Table VI).

\section{Discussion}

It has been shown that one of the most important factors in the success of tympanoplasty is the size and site of the perforation. ${ }^{1,2}$ The success rate of repairing small and posterior perforations can be higher than $90 \%$ but the success rate dropped to $67 \%$ in the repair of anterior and subtotal perforations. ${ }^{8}$

Using flourescein dye, Applebaum and Deutsch demonstrated that the anterior tympanic membrane is less vascular than the posterior part. Because of this reduced vascularity, there is greater risk that epithelialisation and healing may not occur in the anterior part prior to necrosis and re-absorption of the graft. In this area of the ear drum, there is also a problem with fixation of the fascia graft. ${ }^{3}$

One of the main problems in subtotal perforation is the lack of anchorage and support for the graft, especially with the underlay technique. It has been stated that the graft acts as a scaffold for the migration of squamous epithelium and mucosa. Hence persistence of the graft in correct position is more critical to the successful closure of the large perforation than small one.
Schuknecht (1976) believes that surgical technique is most important factor in the success of tympanoplasty especially for anterior and subtotal perforations. ${ }^{9}$ Two classic methods for reconstruction of TM perforation have been medial and lateral technique. Each technique has its advantages and disadvantages..$^{10,11}$

In medial technique, the graft is placed medial to the drum remnant after excision of the edges of the perforation. It is simple and easy to perform particularly when perforation is small. There is no anterior sulcus blunting or lateralization of graft. But there is a chance of reduction in the middle ear space. Moreover medial technique is not suitable for subtotal or total perforations. ${ }^{12-16}$

In lateral technique the epithelium of the drum remnant is elevated from the fibrous layer, this usually being done in continuity with the flaps made from skin after giving the circumferential incision. Advantages of this technique are visualization of anterior meatal skin is usually complete, which is important in cases where the perforation reaches the anterior annulus; the middle ear space is not compromised as the graft is applied outside the existing layer of middle ear mucosa. Although the lateral technique has a higher success rate for the reconstruction of anterior or subtotal TM perforations, serious complications may occur. Disadvantages are squamous cyst formation from remnants of epithelium left behind the skin flaps; blunting of the anterior sulcus due to accumulation and organization of blood deep to skin graft or due to lateral deposition of the fascia graft over the anterior canal wall; lateralization of the graft which is considered to be a continuation of a process of blunting. In the later postoperative period the graft may become lateralized due to contractile tightening of the graft tissue. ${ }^{12-16}$ In lateralization, the TM loses contact with the conductive mechanism of the middle ear resulting in hearing loss.

Various techniques have been described to overcome the problems. Stage J and Back-Pederson (1992), presented a study in which the graft was placed lateral to the handle of malleus in underlay tympanoplasty. ${ }^{17}$ Kartush et.al. (2002) introduce the term over-underlay tympanoplasty. It was a contribution of two classical techniques; in which the graft is placed lateral to the handle of malleus, and under the drum remnants and 
annulus. ${ }^{18}$ Farrior (1989) in the management of anterior and subtotal tympanic membrane perforations described the sandwich graft tympanoplasty which uses both an internal and external layer of areolar fascia, plus immediate epithelial coverage and reported a $98 \%$ success rate. ${ }^{4}$ Weider (1981) reported a 99\% success rate with the use of the William's microclip to secure the fascia graft. ${ }^{5}$ Cody DT and Taylor WF (1973) reported the use of double fascia grafts, one medial and one lateral to the drum remnant, which yielded a higher rate of successful perforation closure than using single layer grafting techniques. ${ }^{19}$ Hung et.al. (2004), used anterosuperior anchoring technique and reported success rate between $85.7 \%$ to $100 \% .{ }^{20}$ Gerlinger et.al. (2006) described anterior anchoring technique combining the anterior "pull-back" method and reported $100 \%$ graft take up rate. ${ }^{21}$

Jung and Park (2005) described medio-lateral tympanoplasty for anterior or subtotal tympanic membrane perforation and reported $97 \%$ success rate. ${ }^{7}$ In 2009 Jung et. al. presented a comparative study between medial and medio-lateral graft tympanoplasty and reported a success rate of $97 \%$ using the mediolateral technique. ${ }^{22}$ In this technique, the fascia graft is placed medial to the handle of malleus and posterior half of the tympanic membrane perforation but lateral to the anterior half of the perforation. This method is a hybrid of the medial and lateral graft techniques that takes advantages of both methods. The advantages include prevention of anterior fall away of the fascia; stability of the graft; prevention of lateralisation of graft; better blood supply because anterior canal skin is rotated as a rotational flap rather than free graft. Jung \& Park $^{7}$ (2005) and Jung et.al. ${ }^{22}$ (2009) reported no case of lateralization.

In the present study age group ranged from 15-50 years; patients younger than 15 years were excluded from the study. This age group was selected due to its likelihood of their proper and regular follow up as well as to rule out failure of graft acceptance due to repeated upper respiratory tract infections as seen in children, and reduced healing tendency of the elderly. More-over presbycusis tends blur the auditory functions in higher age group.

In the present study maximum number of patients was male $(55 \%)$ and females comprises of $45 \%$ of cases with a male to female ratio of $1.2: 1$. This is because males in villages are usually in the habit of taking bath in the ponds and are more liable to suffer from COM as compared to females. Also males seek medical advice much more readily than females, as they have more outdoor life and have more interaction with society.

All the ears were free from active discharge at the time of surgery though there was history of ear discharge in the past. However, some authors have reported good results of tympanoplasty in wet ears. ${ }^{23}$

No correlation was observed between the bilateral involvement and the successful outcome of tympanoplasty in the present study. This is in accordance with the study done by Smyth et.al. ${ }^{24}$

The overall graft uptake rate in this study was $95 \%$ in medio-lateral technique compared to $80 \%$ of medial technique. These results are in accordance to earlier studies by Jung and Park and Jung et.al. ${ }^{7,22}$ There was no case of lateralization in the present study like previous studies.

\section{Conclusion}

The medio-lateral graft method has been developed and used for reconstruction of large central or subtotal TM perforation. It takes advantage of both the medial and lateral grafting methods while avoiding their pitfalls. A high success rate of $95 \%$ similar to other studies provides support in this favour.

\section{References}

1. Sade J, Berco E, Brown M, Weinberg J, Auraham S. Myringoplasty - short and long term results in a training program. J Laryngol Otol. 1981; 95:635-65

2. Booth JB. Myringoplasty : the reasons of failure. J Laryngol Otol. 1974; 88:1223-36

3. Applebaum EL, Deutsch EC. An endoscopic method of tympanic membrane fluorescein angiography. Ann Otol Rhinol Laryngol. 1986; 99:439-43

4. Farrior JB. Sandwich graft tympanoplasty: experience, results, and complications, Laryngoscope 1989; 99:213-7

5. Weider DJ. Use of the Williams microclip in various aspects of tymanoplastic surgery, Laryngoscope 1981; 91 (12):2106-25

6. Kartush JM, Michaelides EM, Becvarovski Z, LaRouere MJ. 
Over-under tympanoplasty, Laryngoscope 2002; 112 (5):802-7

7. Park SK, Jung TT. Mediolateral graft tympanoplasty for anterior or subtotal tympanic membrane perforation. Otolaryngol Head Neck Surg. 2005; 132:532-6

8. Bhat NA, De R. Retrospective analysis of surgical outcome, symptoms changes and hearing improvement following myringoplasty. J Laryngol Otol. 2000; 29:229-32

9. Schuknecht HF. Myringoplasty. Clin Otolaryngol. 1976; 2:5365

10. Rizer FM. Overlay versus underlay tympanoplasty part I: historical review of the literature, part II: the study. Laryngoscope 1997; 107:26-36

11. Wehrs RE. Grafting technique. Otolaryngol Clin North Am. 1999; 32:448-55

12. Glasscock ME. Tympanic membrane grafting with fascia overlay vs underlay technique. Laryngoscope 1973; 83:754-70

13. Doyle PJ, Schleuning AJ, Echevarria J. Tympanoplasty: should grafts be placed medial or lateral to the tympanic membrane. Laryngoscope 1972; 82:1425-30

14. Mendel L, Kuylenstierna RA. Clinical comparison of results of two different methods of closing tympanic membrane perforations. J Laryngol Otol. 1985; 99:339-42

15. Packer P, Mackendrick A, Solar M. Whats best in myringoplasty: underlay or overlay, dura or fascia? J Laryngol Otol. 1982; 96:25-41
16. Gulati SP, Sachdeva OP, Thakral A, Jain P, Sachdeva A. A comparative evalution of onlay and inlay techniques in myringoplasty. The Indian Practitioner 1997; 50:123-4

17. Stage J, Bak-Pederson K. Underlay tympanoplasty with the graft lateral to the malleus handle. Otolaryngol Clin North Am. 1992; 17:6-9

18. Kartush JM, Michaelides EM, Becvarovski Z et al. Over-under tympanoplasty. Laryngoscope 2002; 112:802-7

19. Cody DT, Taylor WF. Tympanoplasty: long term results. Ann Otol Rhinol Laryngol. 1973; 82:538-47

20. Hung T, Knight JR, Sankar V. Anterosuperior anchoring myringoplasty technique for anterior and subtotal perforations. Clin Otolaryngol. 2004; 29:210-14

21. Gerlinger I, Rath G, Szanyi I, Pytel J. Myringoplasty for anterior and subtotal perforation using KTP-532 laser. Eur Arch Otorhinolarngol. 2006; 263:816-19

22. Jung T, Kim YH, Kim YH, Park SK, Martin D. Medial or mediolateral graft tympanoplasty for repair of tympanic membrane perforation. Int J Pediatr Otorhinolaryngol. 2009; 73:941-3

23. Nagle SK, Jagade MV, Gandhi SR, Pawar PV. Comparative study of outcome of type I tympanoplasty in dry and wet ear. Indian J Otolaryngol Head Neck Surg. 2009; 61:138-40

24. Smyth GDL. Tympanic reconstruction: Fifteen year report on tympanoplasty Part-II. J Laryngol Otol.1967; 90:713-41. 